\title{
Sub-mm maser VLBI: how do stellar winds break free from the star's gravity?
}

\author{
A.M.S. Richards ${ }^{* a}$ A. Baudry ${ }^{b, c}$ S. Etoka ${ }^{d}$ E.A. Humphreys ${ }^{e}$ C.M.V. Impellizeri ${ }^{f, g}$ H.J. \\ van Langevelde ${ }^{h, i}$ and W. Vlemmings ${ }^{j}$ \\ ${ }^{a}$ Jodrell Bank Centre for Astrophysics, School of Physics and Astronomy, Univ. of Manchester, UK \\ ${ }^{b}$ Univ. Bordeaux, LAB, UMR 5804, F-33270 Floirac, France \\ ${ }^{c}$ CNRS, LAB, UMR 5804, F-33270 Floirac, France \\ ${ }^{d}$ Hamburger Sternwarte, Univ. of Hamburg, D-21029 Hamburg, Germany \\ ${ }^{e}$ European Southern Observatory, Garching, Germany \\ ${ }^{f}$ NRAO, 520 Edgemont Road, Charlottesville, VA22903, USA \\ ${ }^{\mathrm{J}}$ Joint ALMA Observatory/ESO, Santiago, Chile \\ ${ }^{h}$ The Joint Institute for VLBI in Europe, Dwingeloo, the Netherlands \\ ${ }^{i}$ Sterrewacht Leiden, Leiden Univ., the Netherlands \\ ${ }^{j}$ Dept. of Earth and Space Sciences, Chalmers Univ. of Technology, Onsala Space Observatory, \\ Sweden \\ amsrejb.man.ac.uk
}

\begin{abstract}
Sub-mm VLBI with ALMA will be a superb tool for imaging masers, as well as its capabilities as the "Event Horizon Telescope". Individual masing clouds will be resolved in multiple velocity channels, providing both beamed and unbeamed sizes. The relationship between beaming and intensity can then be used to distinguish between steady or turbulent and shocked outflows. Sub-au resolution will also confirm which transitions are co-spatial or segregated, compared with maser models predicting the required small-scale variations in density, temperature, velocity structure and radiation field. This will be aided further by micro-arcsec $\mathrm{cm}$-wave maser results which are already starting to appear from RadioAstron. ALMA by itself will resolve the overall structure and differing extents of the various maser species, along with thermal lines, dust and continuum. Science verification data has already provided the first-ever resolved images of sub-mm water masers and continuum around an evolved star (VY CMa), showing that the high excitation lines are surprisingly extended. This requires local heating, maybe shocks, at many tens of stellar radii. These lines straddle the dust formation zone and future higher resolution observations will also resolve dust clumps and measure their acceleration. Such studies are vital to understanding how material ejected from cool stars breaks free from the stellar gravity, and the role of dust in this process.
\end{abstract}

12th European VLBI Network Symposium and Users Meeting,

7-10 October 2014

Cagliari, Italy

${ }^{*}$ Speaker. 

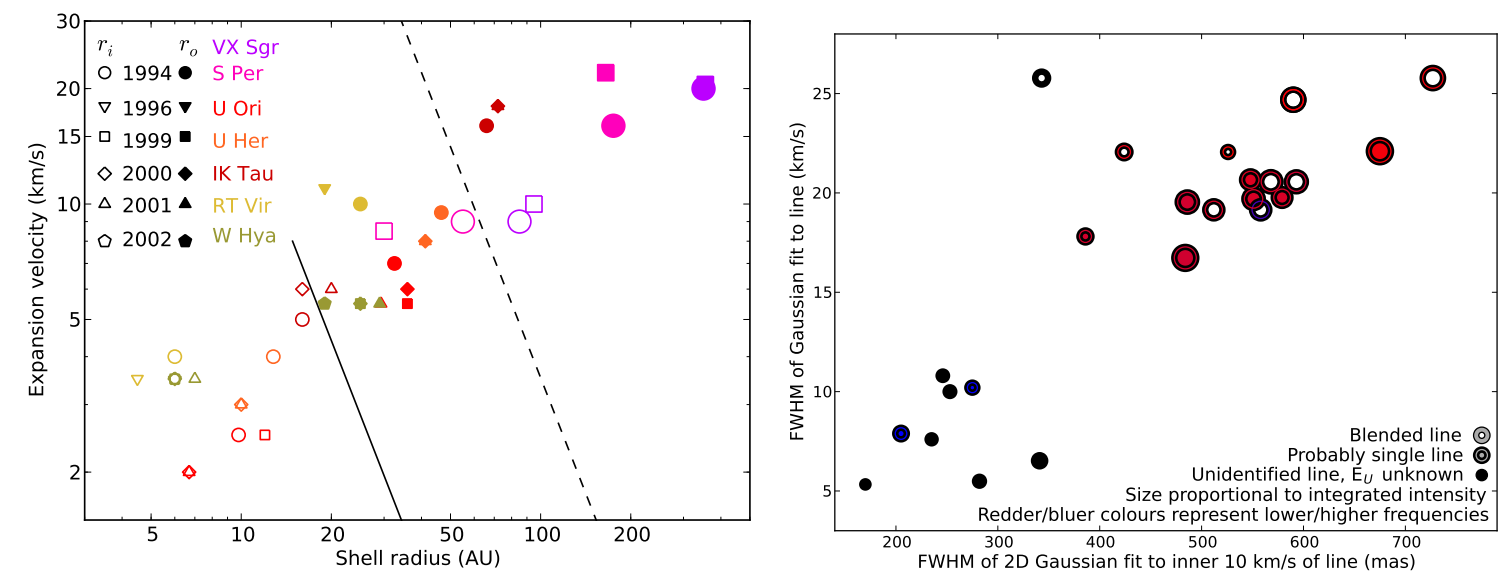

Figure 1: Expansion velocity as a function of distance from the star. (Left) Inner and outer $22 \mathrm{GHz}$ maser shell limits for 2 RSG and 5 AGB stars at different epochs (26). The solid and dashed lines show the escape velocities for AGB and RSG stars, respectively. (Right) Extent of thermal lines around C star CW Leo (11).

\section{How do RSG and AGB stars lose mass?}

Cool $(T \lesssim 3200 \mathrm{~K})$ M-type stars have mass loss rates $\lesssim 10^{-4} \mathrm{M}_{\odot} \mathrm{yr}^{-1}$, ejecting $\sim 80 \%$ of the dust mass in the ISM in a chemically-enriched wind. Asymptotic giant branch (AGB) stars, masses $M<8 \mathrm{M}_{\odot}$, have typical radii $R_{\star} \sim 1$ au, whilst more massive red supergiants (RSG) are up to $10 \times$ larger. These stars are deeply variable with periods of order a year or more and the basic model for mass loss involves levitation of the stellar surface by pulsations (3) until enough dust forms (at $\sim 5-7 R_{\star}$ ) for radiation pressure to accelerate the wind away from the star.

This picture is generally supported by observations but it is not the whole story. There are a number of suggestions for how material is ejected from the stellar surface, involving convection cells, star spots and magnetic effects as well as pulsation. There is no direct relationship between mass loss rate and pulsation amplitude as deduced from optical variability. For example, $\mathrm{SiO}$ masers show evidence for both infall and outflow in the pulsation-dominated regions at $\lesssim 5$ $R_{\star}$ with different authors claiming ballistic motion (16) or clumps being directed along (or dragging) magnetic field lines (17). The transition to steady outflow is controversial, at least for O-rich stars, since the models of (35) suggested that grain growth was insufficient. Some observations (23) and models (1); (2) provide potential mechanisms but these have not yet been widely observationally confirmed. A final conundrum is how spherical main-sequence stars, which (if solitary) lose detectable surface rotation on the AGB/RSG, eventually give rise to often-asymmetric planetary nebulae/supernova remnants. It seems intuitive that there is a connection with the observed, stellar-centred magnetic field, but the origin of such a field poses its own problems.

\section{Clumpy mass loss}

Milli-arcsec scale resolution (e.g. EVN for 1.6-GHz OH masers, $e$-MERLIN for 22- $\mathrm{GHz} \mathrm{H}_{2} \mathrm{O}$ masers) shows that mass loss is concentrated in clumps. The $\mathrm{H}_{2} \mathrm{O} 22-\mathrm{GHz}$ shell $\left(\sim 5-50 R_{\star}\right)$ encloses tens - few 100 clouds with a filling factor $\lesssim 1 \%$ which nonetheless contain between $20 \%$ 

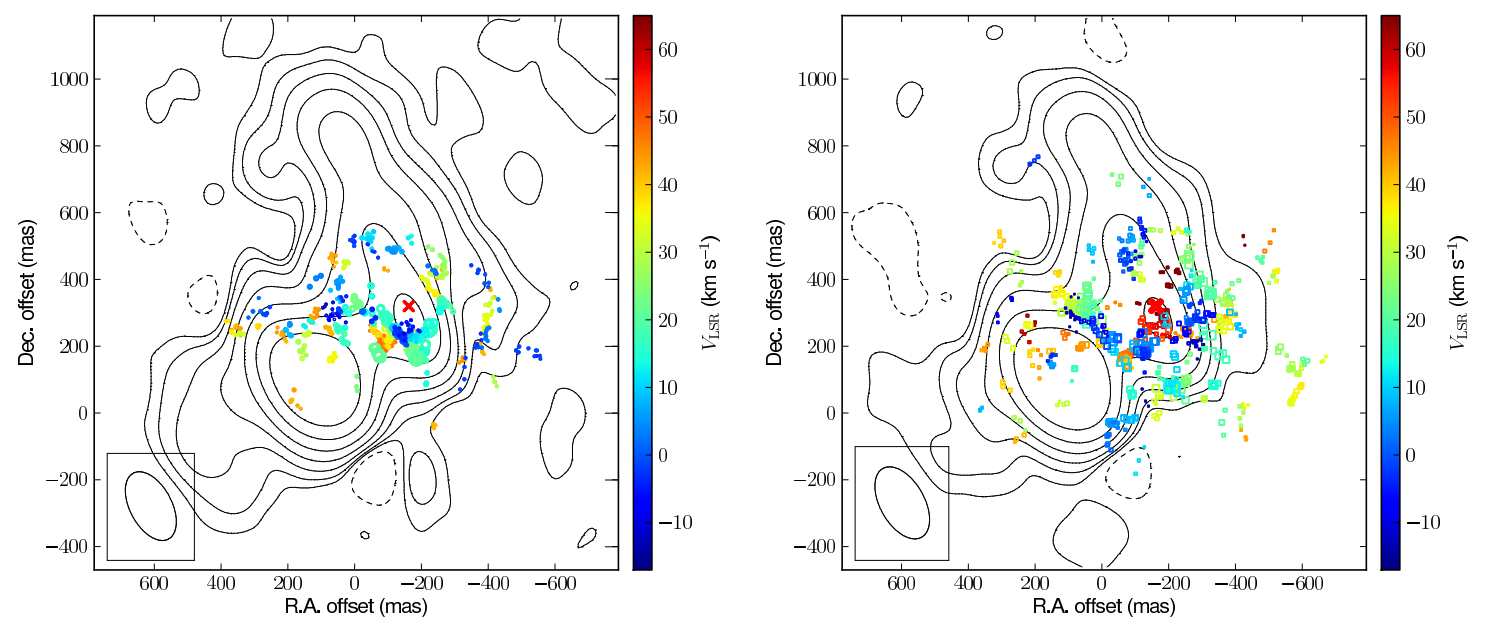

Figure 2: VY CMa $321 \mathrm{GHz}$ (left) and $325 \mathrm{GHz}$ (right) water maser positions over continuum contours, lowest levels at $(-1,1,2,4,8,16,32) \times 1(2) \mathrm{mJy} \mathrm{beam}^{-1}$ at $321(325) \mathrm{GHz}$. Maser symbol size proportional to $\sqrt{\text { flux density, }}$ coloured according to velocity. The red cross marks the star/centre of expansion $\mathbf{V Y}$.

Table 1: $\mathrm{H}_{2} \mathrm{O}$ masers. $v_{2}$ : vibrational state; spin: ortho/para isomers.

\begin{tabular}{cccrcc}
\hline \hline $\begin{array}{c}\text { Frequency } \\
(\mathrm{GHz})\end{array}$ & $\begin{array}{c}\text { Transition } \\
\left(J_{K a, K c}\right)\end{array}$ & $v_{2}$ & $\begin{array}{r}E_{U} \\
(\mathrm{~K})\end{array}$ & $\begin{array}{c}\text { Spin } \\
(\text { level })\end{array}$ & $\begin{array}{c}\text { Discovery } \\
\text { (reference) }\end{array}$ \\
\hline 22.23508 & $6_{1,6}-5_{2,3}$ & 0 & 643 & $o$ & $(6)$ \\
321.22564 & $10_{2,9}-9_{3,6}$ & 0 & 1862 & $o$ & $(19)$ \\
325.15292 & $5_{1,5}-4_{2,2}$ & 0 & 470 & $p$ & $(18)$ \\
658.00655 & $1_{1,0}-1_{0,1}$ & 1 & 2361 & $o$ & $(20)$ \\
\hline
\end{tabular}

and almost all of the mass. The 22-GHz maser clouds have radii approximately proportional to the radius of the parent star; RSG have larger clouds than AGB stars (28). In fact at $\sim 10 R_{\star}$ the cloud radius is similar to the stellar radius, suggesting a birth size of $5-10 \% R_{\star}$. This is comparable to the relative size of convection cells deduced from comparing VLTI and other optical interferometry with models, e.g. (21), (34), (7).

The size of bright maser spots can be measured with accuracy $\sim$ (beam size)/(signal to noise ratio) and this, compared with the maximum angular extent of the series of components making up the cloud, yields the beaming angle (27). Further evidence for inhomogeneity comes from the VLBI detection of $\mathrm{OH} 1665-$ and $1667-\mathrm{MHz}$ masers interleaving the outer 22-GHz clumps; this implies the presence of cooler, lower density gas. Wind acceleration continues throughout the 22GHz shell, attaining escape velocity, as in Fig. 1(left) . Acceleration at many tens $R_{\star}$ is also implied by Herschel and ALMA results showing increasing thermal line width with decreasing excitation temperature (10), (4) and, when resolved, with increasing angular extent, in C-rich CSE also, see Fig. 1 (right) (11). The collision rate is too slow for significant grain growth, so if increased efficiency of radiation pressure on dust is involved, this must be due to optical depth effects (15) and/or changes in dust surface properties (5). If the wind is homogeneous, the drift velocity of dust with respect to gas would vary, but so far this can only be modelled e.g. (31), not observed directly. 

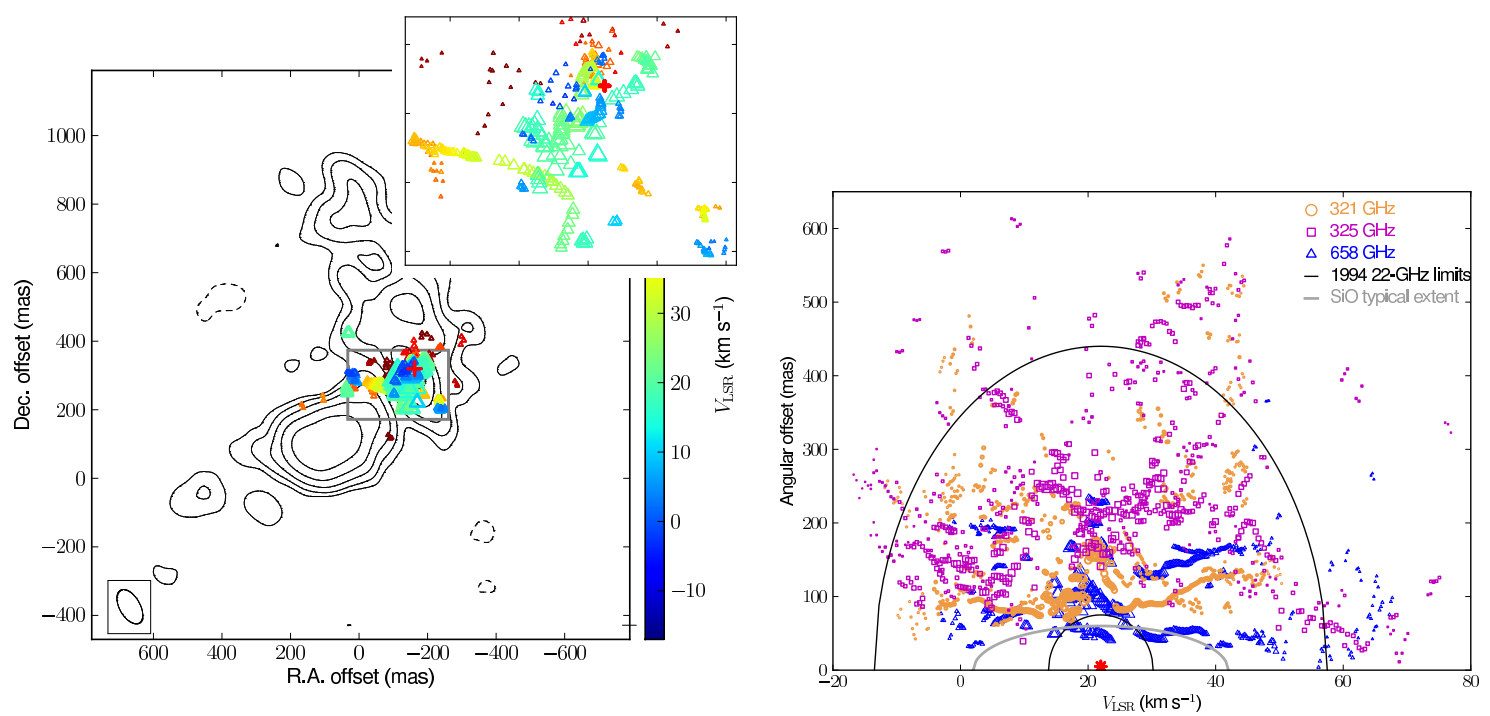

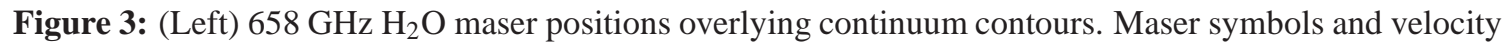
scale as in Fig. 2. The inset shows the region enclosed by the grey box. (Right) Symbols mark sub-mm $\mathrm{H}_{2} \mathrm{O}$ maser component angular separations from $\mathbf{V Y}$ as a function of $V_{\mathrm{LSR}}$. The black lines mark the inner and outer limits of $22 \mathrm{GHz} \mathrm{H}_{2} \mathrm{O}$ masers and the grey line shows the outer limit of $\mathrm{SiO}$ masers (30).

\section{First images of evolved-star sub-mm masers}

ALMA Science Verification observations of the RSG VY CMa were made to test imaging on up to $2.7 \mathrm{~km}$ baselines at around 320 and $660 \mathrm{GHz}$ (up to $\sim 3140 \mathrm{k} \lambda$, restoring beams $\sim 100$ and $\sim 50$ mas). Details are given in (29). The maser transitions observed are listed in Table 1, which gives the upper energy level $E_{U}$ of each transition as well as its frequency and discovery paper. Based on the upper energy levels, densities and temperatures required for maser pumping, it was predicted that the 658,321 and $325-\mathrm{GHz}$ masers would be found at increasing distances from the star (22); (36); (13); (12); (8). Figs. 2 and 3 show that this is qualitatively the case. The masers are expanding away from continuum peak VY, identified as the star. However, closer inspection shows that the 321- and 658-GHz masers are far more extended than predicted. Despite excitation temperatures $\gg 1000 \mathrm{~K}$, they occur at hundreds of au from the star, where the average temperatures are only a few hundred $\mathrm{K}$. The different maser lines are separated on scales of tens au, showing that the environment is homogeneous and they are not co-propagating. One possible explanation is shocks in the wind. Pulsations (speeds $5-7 \mathrm{~km} \mathrm{~s}^{-1},(25)$ ) are very unlikely to penetrate far enough but changes in kinematics due to dust formation (2) could be involved. The 658-GHz masers appear to curve round the SE continuum peak (Fig. 3 and insert), which could indicate a wind collision with a massive dust clump, shown to be cool by (24).

The wind from VY CMa is highly inhomogeneous and has been modelled as a disk plus outflow (9) or ejecta in apparently random directions (14). The elongated dust plumes seen by ALMA show that mass loss towards the $\mathrm{N}$ and $\mathrm{SW}$ has been enhanced for decades, longer than the lifetime of convection cells. At least $17 \%$ of the dust is concentrated in clumps (24). 


\section{Future high-resolution sub-mm imaging}

ALMA in VLBI will resolve the $321 / 325-\mathrm{GHz}$ transitions at $\sim 40 \mu$ as resolution, similar to that of RadioAstron. Ultra-high resolution alone will reveal proper motions of 321- $\mathrm{GHz} \mathrm{H}_{2} \mathrm{O}$ masers close to the star, on similar scales as $\mathrm{SiO}$ masers but probably probing different local conditions. It will allow analysis of the fractal clustering scales of maser spots, which provides diagnostics for incompressible v. compressible turbulence and shocks (33), (32).

Ideally, a range of shorter baseline lengths will be available, from ALMA alone at up to $16 \mathrm{~km}$, providing 5-10 mas resolution for sub-mm masers, and few-hundred $\mathrm{km}$ baselines such as provided by LLAMA (Long Latin American Millimeter Array). This would provide the continuum sensitivity to detect the stars themselves and image tens-mas scale dust clumps, providing comparisons between the expansion velocities of dust and gas and thus direct estimates of the drift velocity. Maser measurements on the scales of individual spots and their parent clouds will show whether the emission peaks are tightly beamed, by approximately spherical, quiescent clouds, or whether brighter emission is more extended, showing the presence of shocks. These results will help to explain the heterogeneity of stellar outflows and whether shock heating is involved in pumping high excitation state masers at large distances from the star.

\section{References}

[1] Bladh, S. \& Höfner, S. 2012, A\&A, 546, A76

[2] Bladh, S., Höfner, S., Nowotny, W., Aringer, B., \& Eriksson, K. 2013, A\&A, 553, A20

[3] Bowen, G. H. 1988, ApJ, 329, 299

[4] Cernicharo, J., Agúndez, M., Kahane, C., et al. 2011, A\&A, 529, L3

[5] Chapman, J. M. \& Cohen, R. J. 1986, MNRAS, 220, 513

[6] Cheung, A.C., Rank, D.M., Townes, C.H., Thornton, D.D., \& Welch, W.J. 1969, Nat, 221, 626

[7] Chiavassa, A., Freytag, B., Masseron, T., \& Plez, B. 2011, A\&A, 535, A22

[8] Daniel, F. \& Cernicharo, J. 2013, A\&A, 553, A70

[9] Decin, L., Hony, S., de Koter, A., et al. 2006, A\&A, 456, 549

[10] Decin, L., Justtanont, K., De Beck, E., et al. 2010, A\&A, 521, L4

[11] Decin, L., Richards, A. M. S., Neufeld, D., et al. 2015, A\&A, 574, A5

[12] Gray, M. D. 2012, Maser Sources in Astrophysics (Cambridge University Press)

[13] Humphreys, E. M. L., Yates, J. A., Gray, M. D., Field, D., \& Bowen, G. H. 2001, A\&A, 379, 501

[14] Humphreys, R. M., Helton, L. A., \& Jones, T. J. 2007, AJ, 133, 2716 
[15] Ivezić, Z. \& Elitzur, M. 2010, MNRAS, 404, 1415

[16] Kemball, A. J., Diamond, P. J., Richter, L., Gonidakis, I., \& Xue, R. 2011, ApJ, 743, 69

[17] Matsumoto, N., Omodaka, T., Imai, H., et al. 2008, PASJ, 60, 1039

[18] Menten, K. M. \& Melnick, G. J. 1991, ApJ, 377, 647

[19] Menten, K. M., Melnick, G. J., \& Phillips, T. G. 1991, ApJ, 350, L41

[20] Menten, K. M. \& Young, K. 1995, ApJ, 450, L67

[21] Montargès, M., Kervella, P., Perrin, G., et al. 2014, A\&A, 572, A17

[22] Neufeld, D. A. \& Melnick, G. J. 1991, ApJ, 368, 215

[23] Norris, B. R. M., Tuthill, P. G., Ireland, M. J., et al. 2012, Nat., 484, 220

[24] O’Gorman, E., Vlemmings, W., Richards, A. M. S., et al. 2015, A\&A, 573, L1

[25] Reid, M. J. \& Menten, K. M. 1997, ApJ, 476, 327

[26] Richards, A. M. S., Davis, R. J., Decin, L., et al. 2013, MNRAS, 432, L61

[27] Richards, A. M. S., Elitzur, M., \& Yates, J. A. 2011, A\&A, 525, A56

[28] Richards, A. M. S., Etoka, S., Gray, M. D., et al. 2012, A\&A, 546, A16

[29] Richards, A. M. S., Impellizzeri, C. M. V., Humphreys, E. M., et al. 2014, A\&A, 572, L9

[30] Richter, L., Kemball, A., \& Jonas, J. 2013, MNRAS, 436, 1708

[31] Sacuto, S., Aringer, B., Hron, J., et al. 2011, A\&A, 525, A42

[32] Silant'ev, N. A., Lekht, E. E., Mendoza-Torres, J. E., \& Rudnitskij, G. M. 2006, A\&A, 453, 989

[33] Strelnitski, V. 2007, in IAU Symposium, Vol. 242, IAU Symposium, 25-31

[34] Wittkowski et al. 2011, A\&A, 532, L7

[35] Woitke, P. 2006, A\&A, 460, L9

[36] Yates, J. A., Field, D., \& Gray, M. D. 1997, MNRAS, 285, 383 